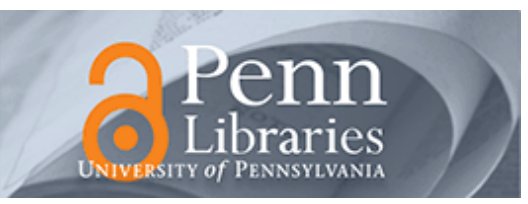

University of Pennsylvania

ScholarlyCommons

March 2005

\title{
Can Bluetooth Succeed as a Large-Scale Ad Hoc Networking Technology?
}

\author{
Evangelos Vergetis \\ University of Pennsylvania \\ Roch A. Guérin \\ University of Pennsylvania, guerin@acm.org \\ Saswati Sarkar \\ University of Pennsylvania, swati@seas.upenn.edu \\ Jacob Rank \\ University of Pennsylvania
}

Follow this and additional works at: https://repository.upenn.edu/ese_papers

\section{Recommended Citation}

Evangelos Vergetis, Roch A. Guérin, Saswati Sarkar, and Jacob Rank, "Can Bluetooth Succeed as a LargeScale Ad Hoc Networking Technology?", . March 2005.

Copyright 2005 IEEE. Reprinted from IEEE Journal on Selected Areas in Communications, Volume 23, Issue 3, March 2005, pages 644-656.

Publisher URL: http://ieeexplore.ieee.org/xpl/tocresult.jsp?isNumber=30451\&page=1

This material is posted here with permission of the IEEE. Such permission of the IEEE does not in any way imply IEEE endorsement of any of the University of Pennsylvania's products or services. Internal or personal use of this material is permitted. However, permission to reprint/republish this material for advertising or promotional purposes or for creating new collective works for resale or redistribution must be obtained from the IEEE by writing to pubs-permissions@ieee.org. By choosing to view this document, you agree to all provisions of the copyright laws protecting it.

This paper is posted at ScholarlyCommons. https://repository.upenn.edu/ese_papers/75

For more information, please contact repository@pobox.upenn.edu. 


\title{
Can Bluetooth Succeed as a Large-Scale Ad Hoc Networking Technology?
}

\author{
Abstract \\ We investigate issues that Bluetooth may face in evolving from a simple wire replacement to a large-scale \\ ad hoc networking technology. We do so by examining the efficacy of Bluetooth in establishing a \\ connected topology, which is a basic requirement of any networking technology. We demonstrate that \\ Bluetooth experiences some fundamental algorithmic challenges in accomplishing this seemingly simple \\ task. Specifically, deciding whether there exists at least one connected topology that satisfies the \\ Bluetooth constraints is NP-hard. Several implementation problems also arise due to the internal \\ structure of the Bluetooth protocol stack. All these together degrade the performance of the network, or \\ increase the complexity of operation. Given the availability of efficient substitute technologies, Bluetooth's \\ use may end up being limited to small ad hoc networks.

\section{Keywords} \\ Bluetooth, performance, scatternets, topology formation, wireless ad hoc networks

\section{Comments} \\ Copyright 2005 IEEE. Reprinted from IEEE Journal on Selected Areas in Communications, Volume 23, \\ Issue 3, March 2005, pages 644-656. \\ Publisher URL: http://ieeexplore.iee. org/xpl/tocresult.jsp?isNumber=30451\&page=1 \\ This material is posted here with permission of the IEEE. Such permission of the IEEE does not in any way \\ imply IEEE endorsement of any of the University of Pennsylvania's products or services. Internal or \\ personal use of this material is permitted. However, permission to reprint/republish this material for \\ advertising or promotional purposes or for creating new collective works for resale or redistribution must \\ be obtained from the IEEE by writing to pubs-permissions@ieee.org. By choosing to view this document, \\ you agree to all provisions of the copyright laws protecting it.
}




\title{
Can Bluetooth Succeed as a Large-Scale Ad Hoc Networking Technology?
}

\author{
Evangelos Vergetis, Student Member, IEEE, Roch Guérin, Fellow, IEEE, Saswati Sarkar, Member, IEEE, and \\ Jacob Rank
}

\begin{abstract}
We investigate issues that Bluetooth may face in evolving from a simple wire replacement to a large-scale ad hoc networking technology. We do so by examining the efficacy of Bluetooth in establishing a connected topology, which is a basic requirement of any networking technology. We demonstrate that Bluetooth experiences some fundamental algorithmic challenges in accomplishing this seemingly simple task. Specifically, deciding whether there exists at least one connected topology that satisfies the Bluetooth constraints is NP-hard. Several implementation problems also arise due to the internal structure of the Bluetooth protocol stack. All these together degrade the performance of the network, or increase the complexity of operation. Given the availability of efficient substitute technologies, Bluetooth's use may end up being limited to small ad hoc networks.
\end{abstract}

Index Terms-Bluetooth, performance, scatternets, topology formation, wireless ad hoc networks.

\section{INTRODUCTION}

B LUETOOTH, a short-range low-power communication protocol, was initially envisioned as a wire replacement solution. Bluetooth uses a design paradigm that is fundamentally different from that of competing technologies like IEEE 802.11. This motivates an examination of the extent to which Bluetooth can be used in a networking context, and in particular, large ad hoc networks. IEEE 802.11 is a simple distributed protocol, where a node can transmit whenever it senses a free channel. The resulting collisions, however, waste bandwidth and power. On the other hand, Bluetooth is partly distributed and partly centralized. It has a hierarchical organization where the nodes are organized in groups denoted as piconets. In each group, a master node controls the transmissions of other nodes. This local control eliminates collisions and is, therefore, expected to offer high throughput and low power consumption. We, however, demonstrate that this organization introduces significant complexity in establishing a connected topology in large and dynamic ad hoc networks. Given Bluetooth's difficulty in fulfilling the simplest of all networking tasks, that of attaining connectivity, its use is likely to be limited to small ad hoc networks.

Manuscript received October 15, 2003; revised November 1, 2004. This work was supported in part by the National Science Foundation (NSF) under Grant ITR-0085930, Grant ANI-0106984, Grant ANI-9902943, Grant ANI-9906855, and Grant NCR-0238340.

The authors are with the Department of Electrical and Systems Engineering, University of Pennsylvania, Philadelphia, PA 19104-6390 USA (e-mail: vergetis@seas.upenn.edu; guerin@ee.upenn.edu; swati@ee.upenn.edu; jrank@ alumni@upenn.edu).

Digital Object Identifier 10.1109/JSAC.2004.842544
The difference between 802.11 and Bluetooth is analogous to that between Ethernet and token ring. Token ring offered a higher throughput but was more complex. The increase in transmission speeds more than compensated for Ethernet's throughput inefficiency. Although bandwidth constraints are greater in the wireless setting, we believe that the choice between 802.11 and Bluetooth will also be guided by simplicity of operation. This is because operational complexity seriously undermines the operation of large dynamic ad hoc networks as nodes have limited processing power and computations cannot be offloaded to an infrastructure.

We focus on the basic aspect of topology formation as it illustrates the problems that Bluetooth encounters when used as a networking technology. First, we investigate this problem from an algorithmic perspective to gain a basic understanding of its fundamental complexity. In Section II, we describe the technical challenges related to topology formation in ad hoc networks using Bluetooth. Although Bluetooth nodes are functionally equivalent, communications proceed according to a "master-slave" model, with a constraint on the number of slaves that a master can support. This introduces a degree constraint on the resulting topology graph. Furthermore, the topology formation algorithms need to determine which nodes will be masters and appropriately assign slaves to those masters. In Section III, we show that these constraints have a significant impact on connectivity. Specifically, deciding whether there exists at least one connected topology that satisfies the degree constraint of Bluetooth is NP-hard. This explains why forming a Bluetooth topology in a short time while satisfying all the Bluetooth constraints has been a topic of extensive research for several years.

Next, we explore topology formation algorithms of different complexity (Sections IV and V). We present a polynomial complexity topology formation algorithm that, under some simplifying assumptions, yields a connected topology whenever one exists. We then present several heuristics that produce good results when these simplifying assumptions do not hold, including an efficient and natively distributed algorithm. In Section VI, we develop a detailed emulator of the Bluetooth stack, and use it to evaluate the performance of our most promising solution. Our investigation reveals that in spite of several simplifying assumptions that made for a "best case" evaluation, performance and, in particular, the time it takes to form a stable connected topology, is poor and in some cases (large networks) unacceptable. We confirm that this disappointing showing is not specific to our algorithm through a comprehensive comparison with previously proposed algorithms. This comparison helps highlight 


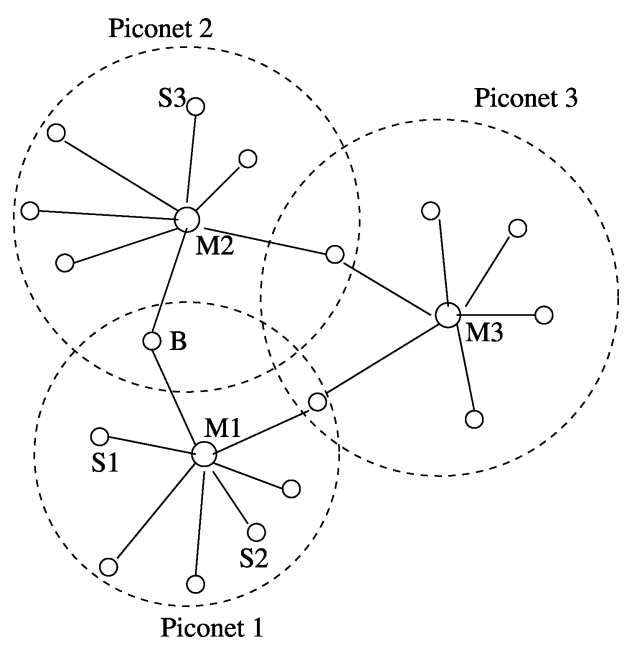

Fig. 1. Example of a Bluetooth topology is illustrated. The nodes are organized into three piconets. The masters of these piconets are $M_{1}, M_{2}$, and $M_{3}$, respectively. The remaining nodes are slave or bridge nodes. Slave nodes $S_{1}$ and $S_{2}$ can communicate via master $M_{1}$. Nodes $S_{1}$ and $S_{3}$ can communicate via master $M_{1}$, bridge $B$, and master $M_{2}$.

key properties and assumptions that are important when evaluating Bluetooth's performance, and leads us to conclude that the performance-minded design choices that were behind Bluetooth's specifications make it difficult, if not impossible, for it to be successful in large-scale ad hoc networks. We examine some related works in Section VII, and conclude in Section VIII.

\section{Challenges AND OBJECtives IN BLUETOOTH TOPOLOGY FORMATION}

We first describe the basic features of the Bluetooth technology that are relevant to topology formation. Bluetooth nodes are organized in small groups called piconets. Every piconet has one "master" node and up to seven "slave" nodes. Refer to Fig. 1 for a sample organization. Slaves in a piconet do not directly communicate with each other, but rely on the master as a transit node. Communication between nodes in different piconets relies on bridge nodes that belong to multiple piconets. A bridge node can only be active in one of the piconets it is connected to at a time. Bluetooth allows different activity states for nodes: active, idle, parked, and sniffing. However, data exchange takes place between two nodes only when both are active, and nodes periodically change their activity state. This combination of flexibility and constraints on which Bluetooth is based raises a number of questions and challenges. We list those that are most relevant to topology formation.

1) How should nodes select their role (master or slave)?

2) Which piconet(s) should a (slave) node join?

3) How many slaves should a master accept (below the specified maximum of seven)?

4) How many piconets should a bridge node belong to?

5) Should a master serve as a slave in other piconets?

When Bluetooth is used as a wire-replacement technology, the above questions have trivial answers. There is only one piconet and one obvious choice for the master, e.g., the computer rather than the keyboard, or the cell phone rather than the head set. The master accepts new slaves as long as the maximum number of seven has not been reached. In ad hoc networks consisting of a small number of piconets, answering the above questions may not incur significant additional complexity. Bluetooth is ideally suited for such simple scenarios. Power consumption is low, and resources can be allocated more efficiently due to the masters' local control.

In a large distributed environment, however, appropriately answering the above questions introduces significant added complexity that can affect network connectivity. ${ }^{1}$ For example, the answer to question 2) depends on how busy the node is, how well connected the topology is, whether the node can play a dual role, etc. Also, answers to the above questions can seriously affect a number of network attributes, e.g., throughput. For example, consider questions 4) and 5). Since a bridge node can only be active in one piconet at a time, the greater the number of piconets to which a node belongs, the poorer the data rate it can provide between them. Thus, it is desirable for a bridge node to be involved in as small a number of piconets as possible, while preserving connectivity. The impact on throughput is compounded when a bridge node is a master in one piconet. This is because all slaves in the piconet are in a communication blackout, when the master is active in other piconets. Thus, it is desirable for a master not to be a slave in other piconets, provided that this does not substantially complicate forming and modifying topologies.

Since nodes select their roles based on local information, efficient algorithms will most likely allow nodes to modify their earlier decisions, e.g., by allowing some slaves to leave one piconet and join another piconet, or by allowing nodes to change their role from slave to master or vice versa. Identifying when and how to allow such changes while preserving the degree constraint or improving connectivity is a challenging task, especially when assuming distributed decisions.

There are several other difficulties above and beyond the development of "clever" topology formation algorithms that introduce additional challenges when using Bluetooth in large ad hoc networks. First, during topology formation, nodes might need to exchange information with each other, and this means establishing a connection where one node will act as the master and the other as a slave. This is easy when neither node belongs to a piconet, but introduces significant complexity when either one or both nodes are engaged in some piconet. For example, a slave and a master can communicate only after they negotiate a time window, called a "sniff" window. In the sniff period, a slave must communicate with or listen to its master. If the slave is not there during this time, then the master terminates the connection (see [1, pp. 163-164]). We demonstrate next through a simple example that determining sniff windows can introduce significant complexity when nodes try to establish a new connection.

Suppose slave $\mathrm{S}$ in piconet $P_{1}$ with master $M_{1}$ is trying to join piconet $P_{2}$ with master $M_{2}$. Let $M_{2}$ have several other slaves, and let the only available sniff window overlap with the sniff window that $\mathrm{S}$ has already established with $M_{1}$. Now, either $\mathrm{S}$ and $M_{1}$ have to negotiate a different sniff window, or $M_{2}$ has

${ }^{1}$ Note that these issues do not arise in 802.11, which highlights the tradeoff associated with different design criteria and their different impact in different environments. 
to move the sniff window of one of its existing slaves. This incurs additional complexity. Now, if neither $M_{1}$ nor the slaves of $M_{2}$ have any other available sniff windows, then the changes in sniff windows can propagate over the whole network! Furthermore, if the master of a piconet is also the slave in some other piconet, determining sniff windows becomes increasingly complex. Thus, topology formation becomes a stumbling block even when we do not consider mobility, or nodes periodically turning their power on or off. Note again that the determination of sniff windows is not an issue in a wire replacement setting, and less likely to be a problem when the number of piconets is small.

The inquiry and page modes used in Bluetooth to allow nodes to discover each other pose yet another challenge. Suppose two nodes A and B receive an "inquiry response" message from node $\mathrm{C}$ at roughly the same time. Then, $\mathrm{A}$ and $\mathrm{B}$ will both page $\mathrm{C}$ repeatedly and their "page" messages will collide. Although this may be solved via randomization, it can introduce a delay in the node discovery process and in the formation of connections.

In conclusion and as we quantify later, by focusing extensively on controlling the use of resources, Bluetooth ends up violating a basic design principle in networking — simplicity of operation - which is critical in large distributed systems. The complexity it introduces in providing connectivity more than offsets any resource optimization capabilities it may afford.

\section{Network Model AND PROBLEM COMPLEXITY}

We formulate next a mathematical model for the system's objectives and constraints. There can be two types of links between any two nodes. One is a physical (layer) link that exists between any pair of nodes that are in communication range of each other. The other is a logical Bluetooth link that exists if the Bluetooth topology establishes an actual communication link between the two nodes. The physical topology graph is determined by the positions and the transmission radii of the nodes, while the logical topology graph is generated by the topology formation algorithm.

The logical topology graph must have certain properties. According to the Bluetooth specification, vertices that will be assigned the role of a master can have a maximum degree ${ }^{2}$ of 7 . For the vertices that will serve as slaves, it is desirable that their degree be kept as small as possible. Regular slave nodes have a degree of only 1 , but bridge nodes have a degree equal to the number of piconets they participate in. Since a bridge node with a degree more than 7 would provide poor data rate between the piconets it connects, we assume that the degree constraint of 7 applies to the bridge (slave) nodes as well. We choose the number 7 as this will give the same degree constraint for master, slave and bridge nodes. The logical topology graph is bipartite ${ }^{3}$ when the desirable condition that a master is not a slave in another piconet holds.

Connectivity is then deemed feasible if there exists a connected $^{4}$ subgraph of the physical topology graph which satisfies the degree constraint (maximum degree of 7). If connectivity is

\footnotetext{
${ }^{2}$ The degree of a vertex is the number of edges originating from the vertex.

${ }^{3} \mathrm{~A}$ bipartite graph is one where the vertex set can be partitioned in two sets such that there is no edge connecting two vertices in the same set.

${ }^{4} \mathrm{~A}$ graph is connected if there is a path between any two nodes.
}

feasible, then we want to construct a connected logical topology graph that satisfies the desired degree constraint. Otherwise, any logical topology graph will consist of "islands" or components, ${ }^{5}$ and we then seek to minimize the number of components in the logical topology graph.

Note that a connected logical subgraph exists if and only if the physical topology graph has a spanning tree ${ }^{6}$ that satisfies the degree constraint of a logical topology graph. This is because a spanning tree of any graph is connected and bipartite [2]. In a spanning tree, the partition that has a maximum degree less than or equal to 7 is chosen as the master set, while the other with a potentially lower maximum degree forms the slave/bridge set.

Let the degree of a spanning tree be the maximum degree of its vertices. A spanning tree with degree less than or equal to 7 exists if and only if the maximum degree of a spanning tree in a graph is upper bounded by 7, and deciding this is NP-hard [3]. Thus, deciding whether connectivity is feasible and constructing a connected logical topology graph which satisfies the desired degree constraint is NP-hard.

Nevertheless, polynomial time algorithms are available in certain practical scenarios, where additional constraints are imposed on the underlying network graph (Section IV-B). Furthermore, we show how those polynomial time algorithms can be extended to provide efficient heuristics in general scenarios (Section IV-C). Many of these algorithms are centralized, but the basic intuition behind them motivates a fully distributed and dynamic approximation (Sections V and VI).

\section{EXPLORING THE RANGE OF POSSIBLE SOLUTIONS}

We explore the range of algorithms that are capable of forming the desired topologies. We start with a naïve algorithm, continue with algorithms for nodes on a plane, and finally present algorithms that operate in three-dimensional (3-D) space.

\section{A. Nä̈ve Algorithm for Topology Formation}

We first consider a naïve algorithm where a node randomly chooses its role as either master or slave [4]. Then, if it is a slave, it accepts every connection request up to the limit of 7 , and if it is a master, it pages slave nodes until it forms seven connections. Here, using the emulator described in Section VI, we quantify how often this algorithm generates a disconnected topology, even when a connected one exists.

When 100 nodes are uniformly placed on a square of size 1 unit and the transmission radius of each node is 0.25 units, the algorithm forms a connected topology with probability 0.39 . However, a connected topology exists with probability 0.86 . Thus, the algorithm fails to form a connected topology about $55 \%$ of the time. We simulated various other combinations of numbers of nodes $(10,25,50$, and 100$)$ and transmission radii $(0.1,0.17,0.25,0.32,0.4,0.5,0.6$, and 0.75 units). The algorithm failed to construct a connected topology in more than

\footnotetext{
${ }^{5} \mathrm{~A}$ component of a graph is a connected subgraph that cannot be expanded any further while retaining connectivity.

${ }^{6} \mathrm{~A}$ spanning tree is a connected subgraph which does not have a cycle and spans all vertices in the graph.
} 
$20 \%$ of the cases. Moreover, in many cases, this failure probability is much higher than 0.5 (see Fig. 2 for the 50 node case). These results motivate us to develop "smarter" topology formation algorithms.

\section{B. Topology Formation Algorithms for Nodes With Identical Power Levels on a Plane}

We now approach the connectivity problem under certain simplifying assumptions, which we describe and justify next. First, we assume that nodes constitute points on a plane. This assumption is justified in several ground-based civilian and military communication networks where the transceivers are at similar heights and there is no air to ground communication. Second, we assume that nodes have the same transmission range $d$. This happens if the propagation conditions are similar throughout the network and nodes have the same maximum transmission power limitation and similar reception capabilities. Now, a physical link exists between any two nodes if and only if their Euclidean distance is upper bounded by $d$.

Under these two assumptions, the connectivity problem becomes of polynomial complexity. The following Lemma provides the cornerstone for designing a simple polynomial complexity, distributed algorithm that generates a connected logical topology whenever connectivity is feasible.

Lemma 1: Connectivity is feasible if and only if the physical topology graph is connected. A minimum weighted spanning tree (MST) in the physical topology graph, with the weight of an edge equaling the Euclidean distance between the nodes, is a connected logical topology graph that satisfies the constraints.

We first present the following result obtained by Monma et al. [5], which we will use in proving this lemma.

Proportion 1: Consider a complete ${ }^{7}$ graph with nodes corresponding to points on a plane and the weight of the edges being the Euclidean distance between them. Any MST in such a graph has degree less than or equal to 6 .

The intuition behind this proposition is provided in Fig. 3.

Proof of Lemma 1: Clearly, a necessary condition for connectivity to be feasible is that the physical topology graph be connected. We will show that this condition is sufficient as well. Assume that the physical topology graph is connected. Consider a new graph formed by adding edges between all pairs of nodes in the physical topology graph. This graph is referred to as the completion of the physical connectivity graph. The weights of the new edges equal the Euclidean distance between the nodes. The physical topology graph is a subgraph of this completion graph consisting of all edges of the completion graph with weight less than $d$. From Proposition 1, the degree of any MST in the completion graph is less than or equal to 6 . Any MST in the physical topology graph is also an MST in the completion graph. This follows from the following facts: 1) all edges in the completion graph with weight less than $d$ belong to the physical topology graph and 2) the physical topology graph is connected. Thus, any MST in the physical topology graph has degree less than or equal to 6 . Therefore, such an MST satisfies the degree constraint, and is a bipartite graph by virtue of being a tree. Hence, any MST in the physical topology

${ }^{7} \mathrm{~A}$ graph is complete if it has edges between any pair of vertices.

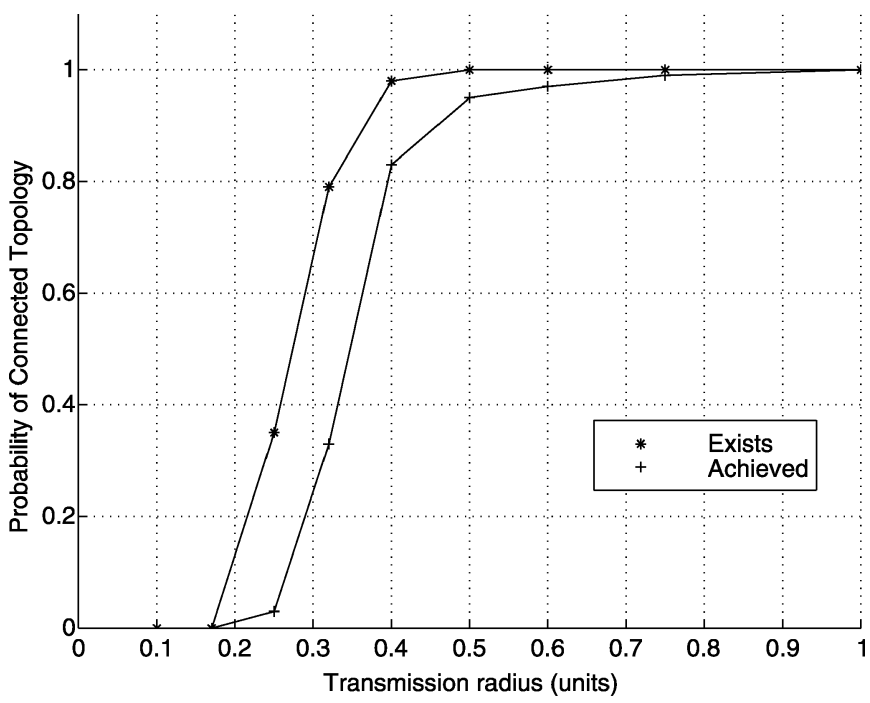

Fig. 2. The line denoted by $*$ corresponds the probability that a connected topology exists. The line denoted by + corresponds to the probability that a connected topology is actually achieved by the naïve algorithm.

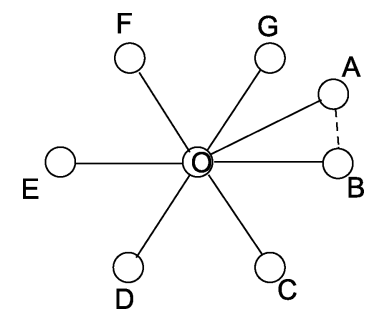

Fig. 3. We explain intuitively why in a complete graph with edge weights equaling the Euclidean distance between the corresponding vertices, the degree of an MST is no more than 6. Consider a complete graph with vertices O, A, $\ldots$, G. Assume that vertex $\mathrm{O}$ in an MST has degree 7. Let its neighbors in the MST be $\{A, \ldots, G\}$. Note that the Euclidean distance between nodes A and B is less than the distance between $(\mathrm{O}, \mathrm{A})$ or $(\mathrm{O}, \mathrm{B})$. Thus, the MST will include the edge $(\mathrm{A}, \mathrm{B})$ rather than $(\mathrm{O}, \mathrm{A})$ or $(\mathrm{O}, \mathrm{B})$.

graph is a connected logical topology graph which satisfies the required constraints.

Next, we consider the case when connectivity is not feasible. This happens only when the physical topology graph is disconnected. The objective in this case is to construct a logical topology graph with the minimum number of components. The following lemma gives the basis for the procedure we follow.

Lemma 2: The subgraph of the physical topology graph consisting of the MSTs in each component of the physical topology graph is a logical topology graph with the minimum number of components.

Proof of Lemma 2: Since a logical topology graph is a subgraph of the physical topology graph, the former has at least as many components as the latter. Thus, the logical topology graph has at least as many components as the subgraph consisting of MSTs in each component of the physical topology graph. It is, thus, sufficient to show that this subgraph satisfies the degree constraint of a logical topology graph. Now, consider each component of the physical topology graph separately. Since each component is connected, then by Lemma 1, the MST in it satisfies the degree constraint of a logical topology graph. Thus, a collection of such disjoint MSTs satisfies the degree constraint of a logical topology graph. 
Lemmas 1 and 2 show that constructing an MST in the physical topology graph will provide a logical topology graph which 1 ) is connected if connectivity is feasible and 2) consists of the minimum number of components if connectivity is not feasible.

Let the physical topology graph have $E$ links and $V$ nodes. Then, an MST can be constructed in a centralized manner with time complexity $O(E \log V)$ [6]. A distributed construction has time complexity $O(V \log V)$ and exchanges $O(V \log V+E)$ messages [7].

The design of a logical topology is not complete without assigning master/slave/bridge roles to the nodes. Since an MST is a bipartite graph, and all nodes have degree less than or equal to 6 , any one partition can be selected as the master set, and the other partition as the slave set. Since we would like to minimize the degree of the bridge nodes, the partition with the smallest degree can be chosen as the slave set.

The MST-based algorithm has, however, some disadvantages. First, if all nodes have low degrees, which is typically going to be the case in an MST, then the end-to-end path between certain nodes may be long, and this causes large end-to-end delay. Thus, the piconet size can be a design parameter. We need to tune the degree of masters to a certain desired value, and the degree of bridges to a different, possibly lower value. The MST algorithm does not allow us to selectively decrease the degrees of the bridges, once the universal degree constraint of 7 is satisfied. We next propose algorithms that can accommodate such a discriminatory treatment, and more importantly, are capable of generating connected topologies when the simplifying assumptions of this section do not hold.

\section{Topology Formation Algorithms for Networks With Nodes in 3-D Space}

We assume that nodes are located in 3-D space and can have different communication ranges. Robins et al. [8] showed that in a 3-D scenario the degree of an MST can be as large as 14, even when all nodes have the same communication range. As a result, enabling an MST-based algorithm to find a connected topology in a 3-D space requires that we relax Bluetooth's constraint to allow up to 15 (instead of 7) active slaves in a piconet. Similarly, when communication ranges are different, even in the two-dimensional (2-D) case, the degree of an MST can exceed 7 (Fig. 4). Hence, the problem needs to be investigated in the framework of a minimum degree spanning tree, which is an NP-hard problem (Section III). We, therefore, investigate heuristics and approximation algorithms.

We next present a topology design procedure that provides a "knob" for separately tuning the degrees of masters and bridges. This is based on an approximation algorithm [minimum degree spanning tree (MDST)] guaranteed to generate a spanning tree with degree at most one more than the minimum possible value in any arbitrary graph (see [9, pp. 272-276]). Thus, MDST generates a connected logical topology in "most" of the instances in which connectivity is feasible. Specifically, the only exception occurs when MDST generates a spanning tree of degree 8 and there exists a connected logical topology with degree 7. MDST starts with any spanning tree, and replaces edges from vertices of high degree with those from vertices of low degree. Refer

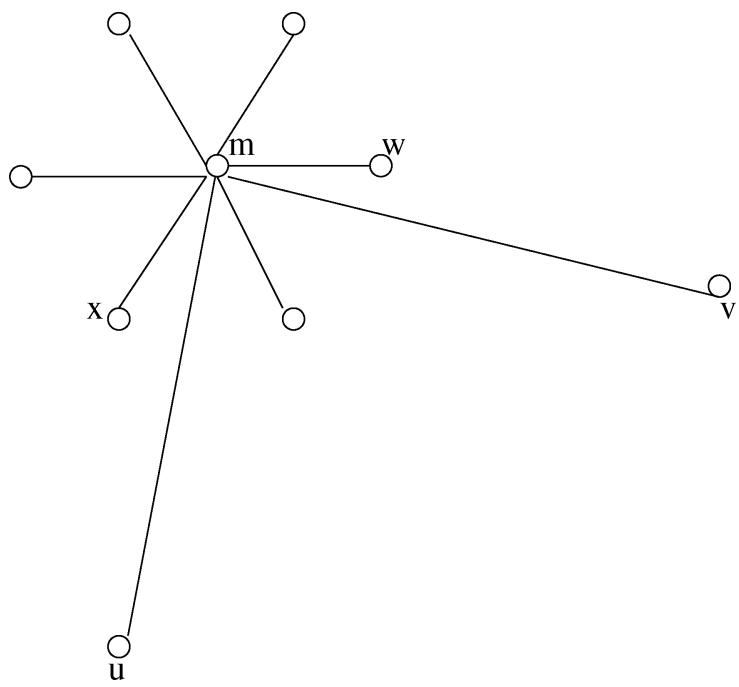

Fig. 4. An example where an MST in a physical topology graph has a degree of 8 . Here, nodes are on a 2-D plane and nodes $\mathrm{m}, \mathrm{v}$, and $\mathrm{u}$ have transmission ranges $100 \mathrm{~m}$, while all other nodes have transmission range $10 \mathrm{~m}$. The solid lines show the MST. Node $\mathrm{m}$ has a degree 8.

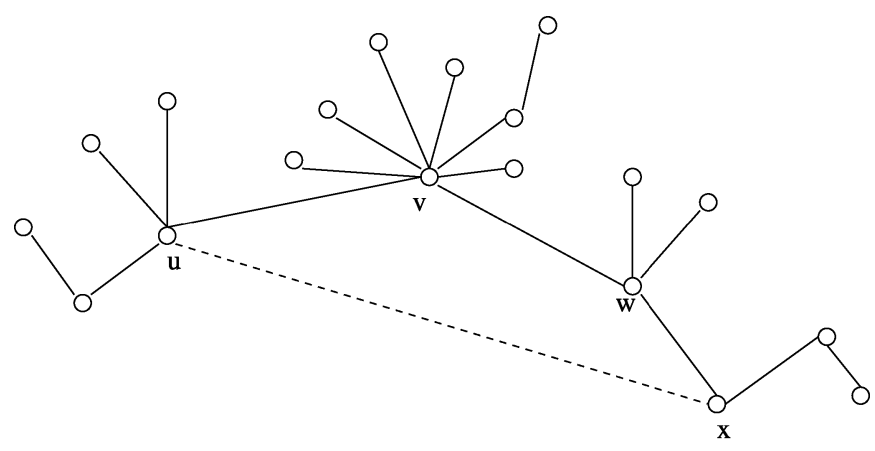

Fig. 5. We explain the operation of the MDST algorithm in this figure. Let the MDST algorithm start with the spanning tree shown in the figure. Node $v$ has degree 8, while all other nodes have degree less than 5. Node $\mathrm{v}$ is marked as "bad," and all other nodes are marked as "good" (since their degrees are less than $d-1=8-1=7$ ). Now, the algorithm considers the cycle generated when edge $(u, x)$ is added to the tree. The degree of node $v$ can now be reduced by including edge $(\mathrm{u}, \mathrm{x})$ in the tree and deleting one of the edges $(\mathrm{u}, \mathrm{v})$ or $(\mathrm{v}, \mathrm{w})$.

to Fig. 5 for an illustrative example. MDST runs in polynomial complexity $O(V E \log V) .^{8}$

We now discuss how to extend MDST to separately control the degrees of the masters and bridges. The goal is to first satisfy a degree constraint of, say, $p$ for all vertices (where $p$ is the desired maximum number of slaves in a piconet and $p \leq 7$ ), and then reduce the maximum degree of the bridges to a desired value, $k$. For this, we use MDST to decrease the degree of a spanning tree generated by breadth first search (BFS) to $p$. Now, edges originating from slaves with degree greater than $k$ are removed from the spanning tree, and replaced by those originating from the masters with degree less than $p$ and slaves with degrees less than $k-1$. The pseudocode for this extension, which is referred to as extended-MDST (E-MDST), follows.

Step 1) Execute MDST on a spanning tree generated by BFS.

${ }^{8}$ More precisely, the run time is $O(V E \alpha(V, E) \log V)$, where $\alpha$ is the inverse of Ackermann's function and grows slowly. For all practical purposes, $\alpha(V, E)$ can be treated as a constant [9]. 
TABLE I

Degree Statistics for the Three Proposed Algorithms. $N$ IS THE Number of Nodes; $M_{a}$ IS The Average Degree of Masters; $M_{m}$ IS THE MAXimum Degree of Masters; $B_{a}$ IS The Average Degree of BRIDGes; $B_{m}$ IS THE MAXIMUM Degree of The BRIDGeS

\begin{tabular}{c||c|c|c|c||c|c|c|c||c|c|r|r}
\hline \multicolumn{1}{c||}{} & \multicolumn{5}{c||}{25} & \multicolumn{5}{c||}{50} & \multicolumn{4}{c}{100} \\
\hline & $M_{a}$ & $M_{m}$ & $B_{a}$ & $B_{m}$ & $M_{a}$ & $M_{m}$ & $B_{a}$ & $B_{m}$ & $M_{a}$ & $M_{m}$ & $B_{a}$ & $B_{m}$ \\
\hline \hline MST & 2.2 & 3 & 2.3 & 3 & 2.3 & 3 & 2.1 & 3 & 2.4 & 4 & 2.3 & 3 \\
\hline MDST & 1.9 & 2 & 2 & 2 & 2 & 2 & 2.1 & 3 & 2 & 2 & 2.1 & 3 \\
\hline E-MDST & 5.9 & 7 & 2.7 & 3 & 6.1 & 7 & 2.4 & 3 & 6.1 & 7 & 2.7 & 3 \\
\hline
\end{tabular}

Step 2) Let MDST output a spanning tree $T$ with degree $p$.

Step 3) The partition with a larger maximum degree is the master set and the other partition is the slave/bridge set. Consider the physical topology graph $G^{\prime}$ with edges between the master and slave sets only.

Step 4) Terminate if the maximum degree $d_{s}$ in $T$ of the slave set is less than or equal to $k$.

Step 5) Mark all master nodes of degree $p$ and all slave nodes of degree $d_{s}$ and $d_{s}-1$ as "bad." A vertex is marked "good" if it is in the "forest" $F=T \backslash$ $\{$ bad vertices $\}$.

Step 6) While there exists an edge $e$ of $G^{\prime}$ that connects two different components of $F$.

a) Consider the cycle $C$ generated by spanning tree $T$ together with $e$.

b) If $C$ has a slave node $w$ of degree $d_{s}$, then denote the edge in $C$ incident on $w$ as $l$, update $T$ by $T \leftarrow T \backslash\{l\} \cup\{e\}$, and go to Step 4$)$.

c) If $C$ does not have any slave node $w$ of degree $d_{s}$, then mark all "bad" vertices in $C$ as good. Update $F$ by combining the components along $C$ and these newly marked vertices into a single component.

Step 7) Output $T$.

We tested MST, MDST, and E-MDST in networks with nodes whose $x$ and $y$ coordinates are uniformly distributed in a square of size 1 unit and $z$ coordinates uniformly distributed between 0 and 0.3 units. We also consider "clustered networks," where the $z$ coordinates of the nodes are selected as above, but the $x$ and $y$ coordinates are clustered. Three square clusters of size 0.4 each are placed randomly in a square of size 1 . A node may belong to one of the three clusters, or it may not belong to any cluster. These four events are equiprobable. If a node belongs to a cluster then its $x$ and $y$ coordinates are uniformly distributed in the corresponding square, otherwise, these are uniformly distributed in the original square of size 1 unit. For each of these two types of node distributions, we evaluate the performance of the algorithms for different number of nodes $(25,50,100)$ and two different transmission radii ( 0.4 and 0.6 units), averaging the results over 100 runs. In all scenarios, node degrees remain well below 7 . Table I shows the results with transmission radius of 0.4 units. The average degree of the masters $\left(M_{a}\right)$ indicate that E-MDST achieves its objective of generating a "bushier" topology, while at the same time attaining a small average degree for the bridges (around 2.7). The results remain similar in the 2-D case and for other node distributions and transmission radii in the $3-\mathrm{D}$ case.

We conclude that all algorithms (MST, MDST, and E-MDST) easily achieve the degree bound imposed by Bluetooth, even in the 3-D case for which MST could possibly yield a degree larger than 7. Hence, the added complexity of MDST over MST does not appear warranted. However, when comparing MST and E-MDST, we see that the latter yields much more compact trees. This motivates considering E-MDST, despite its greater complexity, given that long trees can significantly degrade the network's performance [4].

\section{TOWARDS DISTRIBUTED AND DYNAMIC ALGORITHMS}

In this section, we first illustrate how an MST-based algorithm can be extended to operate in a distributed and dynamic setting. Since this extension is complex, even for an algorithm as simple as MST, we then introduce an algorithm that is inherently distributed and provides similar, albeit somewhat weaker, analytical guarantees.

\section{A. Distributizing an MST-Based Algorithm}

An MST can be constructed by distributed computation at the nodes. Gallager et al. [7] show how Prim's algorithm (see [6, p. 505]) for constructing an MST can be distributized. A node only needs to know an ordering of the weights of its incident edges. In the Bluetooth setting, a node can acquire this knowledge by measuring the signal strength of the synchronization messages sent by its neighbors. If all nodes transmit these messages at the same power level, the signal will be stronger for a neighbor that is closer.

The logical topology needs to be constantly updated due to changes in the physical topology. These changes occur because nodes move and new nodes join and existing nodes leave the system. The spanning tree needs to be updated in response to these topology alterations. See [10] and [11] for efficient algorithms for the dynamic update of MSTs.

The complexity of a distributed and dynamic version of the MST algorithm can, however, be high. In the distributed implementation, nodes are initially singletons, and they gradually merge to form fragments which again merge in order to finally yield an MST. The nodes need to maintain and broadcast a fragment ID, as well as certain information about their outgoing edges in order to decide in a distributed manner which edges to add next [7], [10]. Sniff windows must be established and continuously updated for enabling this exchange of information. But as discussed in Section II, this is a complicated task. Moreover, because of the distributed operation, some nodes will be assigned dual roles. Consider, for example, two fragments $F_{1}$ and $F_{2}$ that are trying to merge by forming a link between nodes $\mathrm{A}$ (which belongs to $F_{1}$ ) and $\mathrm{B}$ (which belongs to $F_{2}$ ). If both $\mathrm{A}$ and $\mathrm{B}$ are masters (or slaves) in their piconets, then forming the link $A B$ means that one of the two nodes will have to assume a 
dual role, or invoke a complex role switching operation for all the nodes in one of the two fragments.

All these complications motivate the consideration of a simpler distributed algorithm that provides weaker analytical guarantees than an MST, but may offer a better tradeoff between performance and complexity.

\section{B. Fully Distributed and Dynamic Algorithm}

We now describe a fully distributed and dynamic algorithm, that results in a topology known as the relative neighborhood graph (RNG) in computational geometry [12]. We refer to this algorithm as the RNG algorithm. RNG adds links as and when they are discovered. Let $|A B|$ denote the Euclidean distance between nodes $A$ and $B$. RNG adds a link between two nodes $A$ and $B$ in the logical topology if and only if $A$ and $B$ are in each others transmission range and $|A B| \leq \max (|B C|,|A C|)$ for any other node $C$ which is in $A$ 's and $B$ 's transmission ranges ("RNG rule"). After RNG has added $A B$, if a node $C$ that violates the above condition is discovered, then RNG deletes $A B$. Fig. 6 illustrates this rule.

We assume that each node knows its neighbors in the physical topology graph $(G)$. A node also knows an ordering among the Euclidean distances between its neighbors from power measurements and subsequent information exchange with its neighbors. Observe that the addition and/or deletion of a link do not affect any other link additions or deletions, and depend only on local information. Hence, there is no need to broadcast any information throughout the graph. Thus, RNG exchanges fewer messages and is simpler than the distributed MST algorithm.

Lemma 3: RNG generates a topology that is a superset of the MST.

Proof of Lemma 3: For simplicity, we assume that there exists a unique MST. Let there exist an edge $A B$ that is chosen by the MST algorithm but not by the RNG algorithm. Thus, there exists a node $C$ such that $|A C|$ and $|B C|$ are less than or equal to $|A B|$. Note that in the MST, at least one of the paths, $A$ to $C$, or $B$ to $C$ must use the link $A B$ (else there is a cycle). Let the path between $B$ and $C$ use this link. Thus, edge $B C$ does not exist (else there is a cycle). Thus, add edge $B C$ to the MST. The earlier path from $C$ to $B$ forms a cycle with edge $B C$, and this cycle contains edge $A B$ (as $A B$ is in the path between $C$ and $B$ by assumption). Remove edge $A B$ from the MST, to construct a spanning tree whose weight is not more than that of the earlier MST (since $|A B| \geq|B C|$ ).

Observe that the above proof holds for any link weights $|A B|$ (not just for Euclidean distances). Thus, the lemma holds for all graphs. The following corollary follows directly from Lemma 3.

Corollary 1: RNG generates a connected logical topology.

Unlike in an MST, there may be multiple paths between any two nodes in an RNG. Thus, an RNG has better connectivity than an MST.

Now, assume that nodes are on a plane and have equal transmission radii. Then, we prove that RNG satisfies the degree constraint of Bluetooth in most cases.

Lemma 4: Let all nodes be on a plane and have equal transmission radii. Let different pairs of nodes have distinct Euclidean distances. Then, the degree of any node in the logical topology generated by RNG is at most 6 .

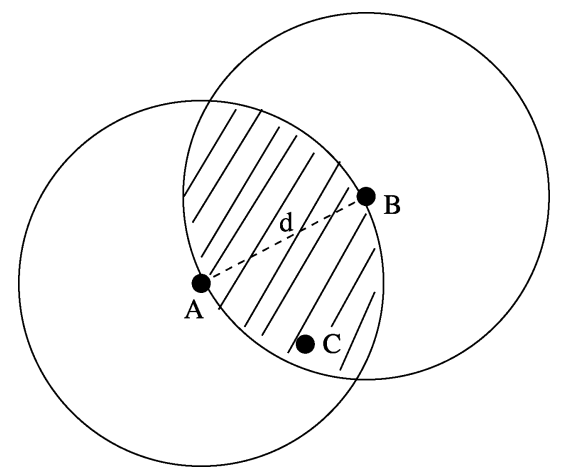

Fig. 6. The two circles in the figure have radii $|A B|$ and centers $A$ and $B$, respectively. RNG would add link $A B$, if there is no other node in the intersection of the circles (shaded area). Link $A B$ is not added in this case as node $\mathrm{C}$ is in the shaded area.

Proof of Lemma 4: Let the degree of a node $O$ in the logical topology generated by RNG exceed 6 (refer to Fig. 3 for an illustration). Then, there exist at least two nodes $A, B$ such that RNG selects edges $O A$ and $O B$, and the angle $A \hat{O} B(\theta)$ is less than or equal to $2 \pi / 6=\pi / 3$. Without loss of generality, let $r_{2}=|O B|<|O A|=r_{1}$. Note that $|O B| \neq$ $|O A|$ by assumption. Let $p=r_{1}-r_{2}$, where by our assumptions $0<p<r_{1}$. From standard geometry, we have that $|A B|=\sqrt{r_{1}^{2}+r_{2}^{2}-2 r_{1} r_{2} \cos \theta}$. Since $\theta \leq \pi / 3$, we have that $\cos \theta \geq 1 / 2$, and using the relation $r_{2}=r_{1}-p$, we get $|A B| \leq \sqrt{r_{1}^{2}-p r_{2}}<r_{1}$. Thus, $|O A|>\max (|A B|,|O B|)$. Since RNG selects edge $O A$ and all nodes have equal transmission ranges, $B$ is in both $A$ 's and in $O$ 's transmission ranges. Thus, RNG will not select edge $O A$, which contradicts our assumption that node $O$ has degree greater than 6 .

Note that different pairs of nodes have distinct Euclidean distances with probability 1 if the $x$ and $y$ coordinates of the nodes are independent continuous random variables with arbitrary density functions. Thus, this condition is satisfied in many practical instances. However, if different pairs of nodes have equal Euclidean distances, then a slight modification of RNG still satisfies the degree bound of 6 [13].

The overall RNG algorithm works as follows. Two nodes $A$ and $B$ that have recently discovered each other, first decide as per the RNG rule ${ }^{9}$ whether to form a connection (i.e., add the link between them to the logical topology). If a connection is to be formed, $A$ and $B$ next decide on their respective roles as masters and/or slaves, according to the following rules. Let $A$ have a higher ID than $B$.

Case 1) When a node powers on, it has an unassigned state.

Case 2) Let $A$ and $B$ have unassigned states when they discover each other. Then, $A$ becomes master, and $B$ becomes a slave in $A$ 's piconet.

Case 3) When one node is unassigned and the other is a master, the unassigned node becomes a slave in the piconet of the master if the piconet has less than seven slaves.

Case 4) When one node is unassigned and the other is a slave, the unassigned node becomes the master of

\footnotetext{
${ }^{9} \mathrm{~A}$ link is not added if one of the incident nodes has a degree of 7 . This situation may arise when nodes are in 3-D space or have unequal transmission ranges.
} 
TABLE II

Evaluation of the RNG Algorithm in a 3-D Clustered Topology. $N$ is the Number of Nodes; $E$ IS THE Number of Edges IN THE RESUlting TOPOlOGY; $D_{i}$ IS THE Average Number of Nodes With Degree $i ; M_{a}$ IS THE AVERAGE Degree OF MASTERs; $B_{a}$ IS THE AVERAGE DEgreE OF BRIDGES; $M / S$ IS THE NuMBER OF Nodes With a Dual Role; $D_{M / S}$ IS The Average Degree of Dual Role Nodes

\begin{tabular}{c|c||c|c|c|c|c||c|c||c|r}
\hline$N$ & $E$ & $D_{1}$ & $D_{2}$ & $D_{3}$ & $D_{4}$ & $D_{5}$ & $M_{a}$ & $B_{a}$ & $M / S$ & $D_{M / S}$ \\
\hline \hline 100 & 117.3 & 9.1 & 50.2 & 37.8 & 2.9 & 0.002 & 2.4 & 2.5 & 16.9 & 2.6 \\
\hline 500 & 616.4 & 24.8 & 236.5 & 219.7 & 18.9 & 0.02 & 2.5 & 2.6 & 93.7 & 2.7 \\
\hline 1000 & 1246.9 & 41.2 & 464.1 & 454.4 & 40.2 & 0.05 & 2.5 & 2.6 & 192.2 & 2.7 \\
\hline
\end{tabular}

a new piconet and the other node joins the piconet as a slave (bridge).

Case 5) If neither $A$ nor $B$ is unassigned, then we consider the following cases separately.
b) If one is a master and the other is a slave in a different piconet, then the slave becomes a bridge between the two piconets.

c) If both are slaves, then $A$ becomes the master and $B$ becomes the slave (bridge).

Thus, some nodes assume dual roles, i.e., they are both a master and a slave [Cases 5a) and 5c)]. This cannot be avoided as the resulting graph is not necessarily bipartite. This situation is not desirable even though the Bluetooth standard allows it. We, therefore, assess the percentage of dual role nodes.

We evaluated the RNG algorithm in the same topologies we considered for MST, MDST, and E-MDST (see Section IV-C). In all scenarios, the degrees of the nodes are below 6 (Table II). The percentage of nodes that have to play a dual role is approximately between $17 \%$ and $19 \%$ of the total number of nodes, but their average degree is still low (around 2.7). The simplicity of the RNG algorithm together with its ability to meet the degree constraint that Bluetooth imposes, make it appealing for practical implementation. However, as we show in the next section, implementing even this simple algorithm in a realistic setting is challenging, and more importantly, its performance may not be adequate.

\section{INVESTIGATING THE RNG ALGORITHM FURTHER}

This section is devoted to investigating the behavior of the RNG algorithm in terms of its ability to form connected topologies in reasonably large ad hoc networks. Our focus is twofold. First, we want to assess RNG's performance in a realistic setting and for a variety of scenarios. Second, we want to compare the RNG algorithm with several existing topology formation algorithms that have been proposed by others. Our main purpose for performing such a comparison is to establish that the conclusions we reach based on the performance of the RNG algorithm, extend to systems using other algorithms as well. Specifically, while be believe that the combination of a native distributed operation, minimum reliance on external information (i.e., only the relative distance between nodes is needed), and strong algorithmic guarantees make the RNG algorithm an ideal candidate for topology formation in Bluetooth, we also want to ensure that this is not achieved at the cost of significantly lower performance (i.e., much larger topology formation times) when compared with other alternatives.

For the purpose of evaluating the performance of the RNG algorithm in a realistic setting, we developed a low level emulator of the Bluetooth protocol stack. The neighbor discovery process, i.e., the inquiry/inquiry_scan and page/page_scan modes, is modeled as described in the Bluetooth specifications [1]. The emulator also includes a limited version of the HCI layer, the interface that allows the control layer to communicate with the lower layers of the stack. The emulator controls the operation of the nodes and gets the information needed for topology formation via specific HCI commands and events (see [1, pp. 373-579]). For example, the emulator computes the distances between the devices from the strength of the received signal, which can be measured by using the Read_RSSI command of HCI. Other commands allow the control layer to instruct nodes to switch between Inquiry and Inquiry_scan modes, create a connection, accept a connection request, etc.

We test the performance of the RNG algorithm in several different scenarios and for different numbers of nodes. In all scenarios, nodes are powered on at random times that are uniformly distributed in an interval between 0-3 s, and node positions are generated as described in Section IV-C. During our initial experiments, we allow nodes to conduct device discovery and topology formation in parallel. When two nodes discover each other, they decide whether to form a logical link as per the RNG rule. However, for simplicity, if two nodes decide to form a logical link, they follow the default Bluetooth behavior, namely, the node performing inquiry becomes the master and the node performing inquiry-scan becomes the slave (or bridge). This differs from the role selection rule specified in Section V-B, which would have required the implementation of a more complex role switching capability. Our goal in following the default Bluetooth behavior is to evaluate the percentage of dual role nodes it would produce and, therefore, better assess the need for implementing a more complex approach that would also affect the time required to form a stable, connected topology.

In addition to basic topology statistics such as average and maximum degrees of nodes of different types and percentage of dual role nodes, we also track several other parameters of interest. The first is the average time $T_{\text {converge }}$ required to converge to the final topology. Convergence to a stable topology is obviously important, as it affects the time taken by routing algorithms to converge and effectively deliver information. We also consider the average time $T_{\text {conn }}$ required to form a connection (measured from the start of Inquiry until the connection is formed) and the average time $T_{\text {enter }}$ a node requires to establish its first link. The latter should be representative of the time it 
TABLE III

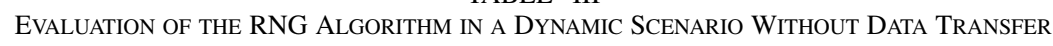

\begin{tabular}{l||c|c|c||c|c|c|c|c|c|c||c}
\hline \multicolumn{1}{l||}{} & \multicolumn{3}{c||}{ Time (sec.) } & \multicolumn{9}{c||}{ Degree Statistics } & \\
\hline $\mathrm{N}$ & $T_{\text {conn }}$ & $T_{\text {enter }}$ & $T_{\text {converge }}$ & Average & $M_{a}$ & $M_{m}$ & $B_{a}$ & $B_{m}$ & $D_{a}$ & $D_{m}$ & $\%$ of Dual \\
\hline \hline 10 & 10 & 11 & 41 & 2.3 & 1.3 & 3 & 2.0 & 3 & 3.4 & 4 & 19 \\
\hline 25 & 12 & 15 & 146 & 2.9 & 1.8 & 3 & 3.6 & 4 & 3.1 & 4 & 44 \\
\hline 50 & 11 & 16 & 223 & 2.8 & 2.1 & 5 & 2.9 & 4 & 3.6 & 5 & 49 \\
\hline 100 & 14 & 21 & 404 & 3.1 & 2.5 & 6 & 2.4 & 5 & 3.2 & 6 & 49 \\
\hline
\end{tabular}

TABLE IV

Evaluation OF THE RNG ALgORITHM IN A DYNAMIC SCENARIO WiTh DATA TRANSFER

\begin{tabular}{l||c|c|c||c|c|c|c|c|c|c||c}
\hline \multicolumn{1}{l||}{} & \multicolumn{3}{c||}{ Time (sec.) } & \multicolumn{6}{c||}{ Degree Statistics } & \\
\hline $\mathbf{N}$ & $T_{\text {conn }}$ & $T_{\text {enter }}$ & $T_{\text {converge }}$ & Average & $M_{a}$ & $M_{m}$ & $B_{a}$ & $B_{m}$ & $D_{a}$ & $D_{m}$ & \% of Dual \\
\hline \hline 10 & 11 & 11 & 61 & 2.4 & 2.1 & 4 & 2.3 & 3 & 2.4 & 3 & 20 \\
\hline 25 & 11 & 15 & 172 & 3.1 & 2.7 & 3 & 2.8 & 4 & 3.7 & 5 & 41 \\
\hline 50 & 13 & 16 & 231 & 2.9 & 2.5 & 4 & 2.3 & 4 & 3.1 & 5 & 47 \\
\hline 100 & 17 & 22 & 434 & 3.2 & 2.3 & 5 & 2.2 & 4 & 3.6 & 5 & 45 \\
\hline
\end{tabular}

would take a new node to connect to an existing network. We consider both the case of nodes spending all their time doing topology formation (Table III), and of nodes that spend $15 \%$ of their time ${ }^{10}$ in data transmission mode (Table IV) during which they are, therefore, not available for topology formation.

In conformance with the results of Section V-B all experiments produce a connected topology (when one exists), and the degrees of all nodes are kept below 7. Due to the device discovery scheme of Bluetooth, the average time to connect $T_{\text {conn }}$ is around $10 \mathrm{~s}$, while a node may have to wait about $20 \mathrm{~s}\left(T_{\text {enter }}\right)$ before entering an existing topology. We next investigate $T_{\text {converge }}$ and observe that even in the ten-node case, it takes about $1 \mathrm{~min}$ to form a stable topology. This time increases to nearly $7 \mathrm{~min}$ when the number of nodes goes up to 100 . When nodes are allowed to spend $15 \%$ of their time in "data transfer" mode, as expected, the time increases even further (Table IV). In addition and consistent with our expectations, the percentage of nodes that assume a dual role is substantially higher in this version of RNG than for the one presented in Section V-B (Table II). This is caused by the difference in the master-slave role selection rules between the two versions of RNG. Allowing role switching as proposed in Section V-B can help lower the number of dual role nodes down to about $20 \%$ (see Table II), which while still high, it may be worth the added complexity.

Those results indicate that even under relatively benign conditions, e.g., no node mobility, homogeneous transmission ranges, etc., and using a simple distributed algorithm such as RNG, forming stable connected topologies in large (of the order of 100 nodes or more) ad hoc Bluetooth networks may take too long to be practical.

Our next step is to confirm those conclusions by comparing the results obtained for the RNG algorithm to data available for other algorithms. Several scatternet formation algorithms have been previously proposed in [15]-[20], and from the results re-

\footnotetext{
${ }^{10}$ This is approximately the time that a slave spends in data transmission if its master has seven slaves. Obviously, masters and bridges will in general spend more time transmitting data. Our model corresponds, therefore, to an optimistic scenario for topology formation.
}

ported on their performance (see Table $\mathrm{VI}^{11}$ ), it appears that they yield significantly lower topology formation times. It is, therefore, important to determine whether this difference is attributable to deficiencies in the RNG algorithm. Upon investigating the characteristics of the above algorithms, and more importantly the operating assumptions used when evaluating them, it appears that there are two main reasons behind the reported differences in performance. The first one is a different model for how node discovery and topology formation are carried out, and the second is a different definition of topology formation time. Specifically, the results are obtained by sequentially carrying out node discovery for a fixed amount of time, and only then initiating the topology formation part. In particular, Basagni et al. [14] assumed in their evaluation of several different algorithms that topology formation was preceded by a fixed 20 -s period of node discovery. In addition, the times reported for topology formation are not always the times until a stable topology has formed, and instead often measure the time it takes to first form a connected topology.

Those two differences, and especially the first one, i.e., sequentially performing the node discovery and topology formation, introduces several significant limitations. First, because device discovery is executed only for a fixed amount of time, not all nodes and links are discovered. Thus, it is possible that some nodes are ultimately unable to communicate. Second, since the full physical topology is not discovered, the analytical guarantees offered by the MST algorithm (Lemma 4), the RNG algorithm (Lemma 1), and several other algorithms, e.g., [15], [17], [19], and [21], no longer hold. Specifically, Basagni et al. [14] show that for a network of 110 nodes, after $20 \mathrm{~s}$ of device discovery, a node only discovers about $88 \%$ of its neighbors. If connectivity depends on the remaining $12 \%$ of the neighbors, then the algorithms will obviously fail to construct a connected topology. Both of those issues are probably not of much significance in small ad hoc networks,

\footnotetext{
${ }^{11}$ Table VI reports the topology formation times for most of those algorithms, as well as additional information regarding their main features. Statistics for LSBS [21], BlueTrees [18], Bluenet [19], and BlueStars [20] were taken from [14] as it provides a detailed comparison of those algorithms. Statistics for the remaining algorithms were taken from the original papers. Note that in several instances, those statistics were obtained using simulators instead of low-level emulators and, thus, the resulting estimates may be somewhat optimistic.
} 
TABLE V

EVAluATION OF THE RNG AlgORITHM: TIME TO FORM THE FIRST CONNECTED TOPOLOGY (USING THE ORIGINAL DEVICE DisCOVERY SCHEME) AND TIME TO FORM A STABLE TOPOLOGY WHEN FOLLOWING THE DEVICE DISCOVERY SCHEME OF [14]

\begin{tabular}{l||c|c}
\hline $\mathrm{N}$ & $T_{\text {connected }}$ (sec.) & $T_{\text {converge }}(\mathrm{sec})$. \\
\hline 10 & 33 & 27 \\
\hline 25 & 63 & 32 \\
\hline 50 & 75 & 36 \\
\hline 100 & 119 & 43 \\
\hline
\end{tabular}

i.e., around ten nodes, where a discovery phase of 20 or even $10 \mathrm{~s}$ will typically be sufficient to discover all nodes, but are likely to result in much more severe problems in large-scale networks. Third but not least, the enforcement of a fixed discovery period that precedes topology formation is difficult, if not impossible, to be implemented in a dynamic environment where nodes power on and off or are mobile.

We believe that the definition of topology formation time and the methodology (parallel and ongoing node discovery and topology formation, progressive power-up of nodes, etc.) used in our experiments with the RNG algorithm, provide for a more realistic and meaningful assessment of topology formation in large Bluetooth ad hoc networks. Nevertheless, in order to allow for a consistent comparison of RNG and the algorithms of Table VI, we perform additional experiments. The first set of experiments still uses our original assumption of parallel and ongoing node discovery and topology formation, but instead of measuring the time it takes for RNG to form a stable topology, we instead track the time $T_{\text {connected }}$ it takes to first form a connected topology. The second set of experiments reproduces the operating conditions of [14], and tracks the time $T_{\text {converge }}$ for RNG to form a topology in such a setting. Those results are shown in Table $\mathrm{V}$, where the column labeled $T_{\text {connected }}$ reports on the first set of experiments, and the column labeled $T_{\text {converge }}$ on the second.

From the values reported for $T_{\text {connected }}$ in Table V, we see that the time taken by RNG to first form a connected topology is much smaller ${ }^{12}$ than the time $T_{\text {converge }}$ (from Tables III and IV) it takes for this topology to stabilize. This is because the "device discovery" process constantly discovers new nodes and links, which occasionally modifies the topology. As discussed earlier, it is difficult for routing to converge until the topology has settled, which may affect reliable data delivery. Thus, we believe that $T_{\text {converge }}$ is a more realistic measure of the time it would take before an ad hoc network forms and becomes operational. Turning to the second column of Table V, we see that when evaluating RNG in a manner consistent with that used to evaluate other algorithms, it yields similar topology formation times. This confirms our initial assessment that the larger topology formation times we had initially observed for RNG are essentially caused by the different operating assumptions we used. As discussed earlier, we believe that our assumptions are more representative of a realistic environment. It should also be pointed out that there are other differences between RNG and some of the algorithms of Table VI. In particular, several of them assume that all nodes are within communication range,

${ }^{12}$ And much closer to the topology formation times of algorithms in Table VI. which essentially eliminates the connectivity constraint but is unlikely to hold in large scale networks.

Finally, we want to point out that there are several additional difficulties in forming stable, connected topologies in large ad hoc networks that neither our Bluetooth emulator nor any of the other simulation results mentioned in Table VI have meaningfully incorporated. One of these factors is the establishment of compatible sniff windows across piconets. Another aspect is node mobility, which would require constant changes to the topology and possibly frequent renegotiations of sniff windows in the different piconets. Both of these are likely to increase topology convergence times, so that the reported figures should probably be considered "best case scenarios," especially for large numbers of nodes. These, together with long topology formation times and the emergence of a relatively large number of dual role nodes, are the bases for our general conclusion that the deployment of Bluetooth as a core technology for building large-scale ad hoc networks is unlikely, especially given the availability of seemingly more suitable alternatives such as 802.11 .

\section{RELATED RESEARCH}

We briefly mention a number of previous works that have been motivated by the ambition to use Bluetooth in ad hoc networks. They span two related areas: 1) assessing the potential of Bluetooth in comparison to other technologies and 2) developing algorithms for forming and maintaining network topologies.

Johansson et al. investigate the suitability of Bluetooth as a networking technology [23], [24]. The authors identify Bluetooth's potential in building personal area networks [23]. They compare Bluetooth to IEEE 802.11 and conclude that in small personal area networks, Bluetooth is better suited than IEEE 802.11. Their conclusions do not apply to large ad hoc networks as they do not consider topology formation and the effects of device discovery.

A few authors have already acknowledged that building Bluetooth-based networks is complex. Basagni et al. [14] identify several problems that the Bluetooth technology gives rise to. They observe that the device discovery process consumes a lot of time, and propose modifications to the Bluetooth standard that may make its operation more efficient. They conclude that forming scatternets is still a formidable task. Liu et al. [25] present an on-demand approach for building a path between Bluetooth devices. However, the delay incurred in their route discovery process is large. Moreover, their results suggest that scatternets face scalability problems. Zheng et al. [26] briefly comment on the complexity of Bluetooth when comparing it to other technologies. Law et al. [15] mention that the problem of collisions of paging messages becomes significant when the number of nodes exceeds 64. Salonidis et al. [27] prove that the average delay involved in synchronizing two nodes is infinite if the nodes rely on a deterministic pattern of alternating between paging and paged modes. This is another issue that is irrelevant when Bluetooth is used as a wire replacement technology, but that is important in a networking context. Chiasserini et al. 
TABLE VI

COMPARISON OF VARIOUS TOPOLOGY FORMATION ALGORITHMS

\begin{tabular}{|c|c|c|c|c|c|c|c|c|}
\hline Algorithm & Ref. & \# Nodes & $\begin{array}{l}\text { Time } \\
\text { (sec.) }\end{array}$ & $\begin{array}{c}\text { Percent } \\
\text { of dual } \\
\text { nodes }\end{array}$ & $\begin{array}{c}\text { Connected } \\
\text { topology } \\
\text { guaranteed? }\end{array}$ & $\begin{array}{c}\text { Degree } \\
\text { constraint } \\
\text { guaranteed? }\end{array}$ & $\begin{array}{l}\text { Devices } \\
\text { within } \\
\text { range? }\end{array}$ & Comments \\
\hline BlueTrees & [18] & 110 & 36 & 50 & Yes & No & Yes & $\begin{array}{l}\text { Needs connected } \\
\text { topology for } \\
\text { "blueroot" election. }\end{array}$ \\
\hline Bluenet & [19] & 110 & 33 & 78 & No & Yes & No & \\
\hline TSF & [16] & 64 & 14 & $\begin{array}{c}\text { See } \\
\text { note }(1)\end{array}$ & Yes & No & Yes & $\begin{array}{c}\text { Elects a } \\
\text { coordinator within } \\
\text { each subtree. }\end{array}$ \\
\hline BlueStars & [20] & 110 & 24 & 22 & Yes & No & No & \\
\hline BlueMesh & [17] & 120 & $\begin{array}{c}\text { See } \\
\text { note }(2)\end{array}$ & $12-17$ & Yes & Yes & No & $\begin{array}{c}\text { Degree constraint } \\
\text { guaranteed only in 2-D. }\end{array}$ \\
\hline $\begin{array}{l}\text { Law } \\
\text { et al. }\end{array}$ & [15] & 128 & 42 & $\begin{array}{c}\text { See } \\
\text { note }(3)\end{array}$ & Yes & $\begin{array}{c}\text { Yes } \\
\text { Note (4) }\end{array}$ & Yes & $\begin{array}{l}\text { Leader election } \\
\text { in each } \\
\text { component. }\end{array}$ \\
\hline LSBS & $\begin{array}{l}{[21]} \\
{[20]} \\
{[14]}\end{array}$ & 110 & 34 & 21 & Yes & Yes & Yes & $\begin{array}{c}\text { Degree constraint } \\
\text { guaranteed only in 2-D. } \\
\text { Needs node locations. }\end{array}$ \\
\hline RNG & [22] & 100 & $\begin{array}{l}43 \\
\text { Note }(5)\end{array}$ & 47 & Yes & Yes & No & $\begin{array}{c}\text { Degree constraint } \\
\text { guaranteed only in 2-D } \\
\text { Needs Euclidean } \\
\text { distance (RSSI). }\end{array}$ \\
\hline
\end{tabular}

[28] consider procedures to handle topology changes in an already existing Bluetooth network. Kallo et al. [29] also consider topology maintenance.

BTCP [27] describes a leader election process to control the topology formation process. It requires all nodes to be in each others transmission range in order to carry out the leader election. This condition is unlikely to hold in general, and also means that the leader election approach of BTCP is not truly a distributed algorithm since all nodes have access to global information to elect a leader. Barrière et al. [30] have proposed a dynamic and distributed algorithm that is capable of achieving not only connectivity, but also of controlling the size of piconets, as well as the desired degrees of masters and slaves. However, it requires that all nodes be capable of communicating with each other, which will often not hold. Finally, Marsan et al. [31] formulate an integer linear program for computing the "optimal" Bluetooth topology. The complexity of the proposed algorithm is, however, high. Furthermore, the integer linear program can only be solved in a centralized manner.

As discussed earlier, several topology formation algorithms have been proposed and evaluated, and Table VI summarizes their main properties and performance. Additional comments and clarification regarding the properties of the different algorithms are provided in the notes that accompany Table VI. None of the distributed algorithms listed in Table VI are guaranteed to produce connected topologies that satisfy the degree constraints of Bluetooth in general settings. This can be explained by our result that satisfying both requirements is an NP-hard problem. Like the MST and RNG algorithms we presented in Sections IV-B and VI, BlueMesh [17] and LSBS [21] satisfy both these requirements only when nodes are on a plane and have equal transmission ranges. In addition, LSBS assumes that each node knows its own and its neighbors' locations. This requires additional hardware, e.g., a GPS receiver and is, therefore, not consistent with Bluetooth's design goal of providing low cost energy-efficient transceivers. By using the relative neighborhood graph structure, which is a subset of the geometric structure (Delauney triangulation) that LSBS uses, the RNG algorithm achieves similar connectivity and degree constraint guarantees as LSBS, but does not require nodes to be location aware.

Notes on Table VI.

1) Tan et al. [16] do not provide statistics on the percentage of nodes that assume a dual role. However, since components (subtrees) merge only from their root nodes, some roots will have to assume a dual role. The nodes can subsequently switch their roles, but switching roles would require network-wide changes.

2) Petrioli et al. [17] mention that on average about four iterations are required to complete the scatternet formation process for 120 nodes. However, there is no information on how much time each iteration takes. Moreover, there is no information on how much time the first phase (topology discovery) of the protocol takes. Given the results of [14], it is likely that each node takes more than $20 \mathrm{~s}$ to discover its one- and two-hop neighbors.

3) Since each leader executes SEEK (i.e., Inquiry) or SCAN (i.e., Inquiry_Scan) using a randomized procedure, some nodes will have dual roles. However, no statistics on the percentage of nodes that assume a dual role are available.

4) The authors focus on the case where all devices are within range. However, in [14, Sec. 8.3, p. 11], they discuss a scenario allowing out of range devices, and mention that in this case the degree constraint is not guaranteed. 
5) For uniform comparison (as in [14]), we report the running time of the algorithm when devices discover each other for $20 \mathrm{~s}$ (Table V).

\section{CONCLUSION}

In this paper, we investigate the feasibility of using Bluetooth as the base communication technology in large-scale ad hoc networks, a task that significantly exceeds its initial scope of a "wire replacement" technology. Our investigation is motivated by Bluetooth's design paradigm that is fundamentally different from that of competing technologies such as IEEE 802.11. We focus on the basic aspect of topology formation as it illustrates the problems that Bluetooth encounters when used as a networking technology. We first investigate this problem from an algorithmic perspective to gain a basic understanding of its fundamental complexity, and establish that deciding whether there exists at least one connected topology that satisfies the degree constraint of Bluetooth is NP-hard. This explains why forming a topology in a short time while satisfying all the Bluetooth constraints has remained elusive even after several years of extensive research. However, we also prove that an MSTbased algorithm is guaranteed to satisfy Bluetooth's constraints under some simplifying assumptions. We also propose several heuristics that satisfy Bluetooth constraints under most conditions and do not rely on those assumptions. Some of these heuristics can differentially control the degrees of masters and slaves, and thereby attain a better delay/throughput tradeoff. These results provide the foundation for an in-depth investigation of Bluetooth's implementation complexity and operational overhead when used as an ad hoc network technology.

For a comprehensive and realistic investigation of Bluetooth's implementation complexity, we designed a detailed low-level emulator of the Bluetooth stack, and used it to examine the convergence time and complexity of a simple, distributed algorithm (RNG) that is capable of satisfying Bluetooth guarantees in most environments. Our findings are that although the algorithm succeeds in forming connected topologies, the time required to generate a stable topology in the presence of a large number of nodes is large enough that it is unlikely to be practical. Furthermore, the presence of a large percentage of dual role nodes substantially impacts the network throughput. These already poor results would only worsen if all the other constraints imposed by the Bluetooth protocol, e.g., sniff window negotiations, handling of node mobility and topology adjustments, etc., were taken into account. Several topology formation algorithms proposed by other authors also perform similarly. As a result, we believe that in spite of the significant attention it has received over the past few years and the many interesting proposals and results it has generated, Bluetooth's inherent complexity as a networking protocol makes it unlikely that it will be widely used in building large ad hoc networks. Nevertheless, it is certainly possible for Bluetooth to be successfully used in building small ad hoc networks, where the issue of topology formation is of much lesser concern.

\section{ACKNOWLEDGMENT}

The authors would like to thank Dr. M. Kodialam, currently at Bell Laboratories, Lucent Technologies, Holmdel, NJ, for directing us to relative neighborhood graphs.

\section{REFERENCES}

[1] Bluetooth SIC, Specification of the Bluetooth system, Version 1.2, Nov. 2003.

[2] F. Harary, Graph Theory. Reading, MA: Addison-Wesley, 1969.

[3] M. R. Gary and D. S. Johnson, Computers and Intractability. San Francisco, CA: Freeman, 1979.

[4] R. Guérin, E. Kim, and S. Sarkar, "Bluetooth technology: Key challenges and initial research," presented at the Commun. Netw. Distrib. Syst. Model Simul. Conf. (CNDS), San Antonio, TX, Jan. 2002.

[5] C. Monma and S. Suri, "Transitions in geometric minimum spanning trees," Discrete Comput. Geometry, vol. 8, no. 3, 1992.

[6] T. Cormen, C. Leiserson, and R. Rivest, Introduction to Algorithms. Cambridge, MA: MIT, 1990.

[7] R. Gallager, P. Humblet, and P. Spira, "A distributed algorithm for minimum-weight spanning trees," ACM Trans. Program. Lang. Syst., vol. 5 , no. 1 , pp. 2-8, Jan. 1983.

[8] G. Robins and J. Salowe, "On the maximum degree of minimum spanning trees," presented at the ACM Symp. Comput. Geometry, New York, Jun. 1994

[9] D. Hochbaum, Approximation Algorithms for NP-Hard Problems. Boston, MA: PWS-Kent, 1995.

[10] C. Cheng, I. Cimet, and S. Kumar, "A protocol to maintain a minimum spanning tree in a dynamic topology," presented at the ACM Symp. Commun. Arch. Prot., Stanford, CA, Aug. 1988.

[11] P. Nárvaez, K.-Y. Siu, and H.-Y. Tzeng, "New dynamic algorithms for shortest path tree computation," IEEE/ACM Trans. Netw., vol. 8, no. 6 , pp. 734-746, Dec. 2000.

[12] G. T. Toussaint, "The relative neighborhood graph of a finite planar set," Pattern Recognition, vol. 12, no. 4, pp. 261-268, 1980.

[13] X. Li, Y. Wang, P. Wan, and O. Frieder, "Localized low weight graph and its applications in wireless ad hoc networks," in Proc. INFOCOM, Hong Kong, China, Mar. 2004, pp. 431-442.

[14] S. Basagni, R. Bruno, G. Mambrini, and C. Petrioli, "Comparative performance evaluation of scatternet formation protocols for networks of Bluetooth devices," Wireless Netw., vol. 10, no. 2, Mar. 2004.

[15] C. Law, A. K. Mehta, and K.-Y. Siu, "A new Bluetooth scatternet formation protocol," Mobile Netw. Apps., vol. 8, no. 5, Oct. 2003.

[16] G. Tan, A. Miu, J. Guttag, and H. Balakrishnan, "An efficient scatternet formation algorithm for dynamic environments," presented at the IASTED Int. Conf. Commun. Comput. Netw., Cambridge, MA, Nov. 2002.

[17] C. Petrioli, S. Basagni, and I. Chlamtae, "BlueMesh: Degree-constrained multihop scatternet formation for Bluetooth networks," Mobile Netw. and Apps., vol. 9, no. 1, Feb. 2004.

[18] G. Záruba, S. Basagni, and I. Chlamtac, "Bluetrees-scatternet formation to enable Bluetooth-based ad hoc networks," in Proc. IEEE Int. Conf. Commun. (ICC), vol. 1, Helsinki, Finland, Jun. 2001, pp. 273-277.

[19] Z. Wang, R. Thomas, and Z. Haas, "Bluenet-A new scatternet formation scheme," presented at the 35th Hawaii Int. Conf. Sys. Sciences (HICSS-35), Big Island, HI, Jan. 2002.

[20] C. Petrioli, S. Basagni, and I. Chlamtac, "Configuring BlueStars: multihop scatternet formation for Bluetooth networks," IEEE Trans. Comput., Special Issue Wireless Internet, vol. 52, no. 6, pp. 779-790, Jun. 2003.

[21] X.-Y. Li, I. Stojmenovic, and Y. Wang, "Partial Delaunay triangulation and degree limited localized Bluetooth scatternet formation," IEEE Trans. Parallel Distrib. Syst., vol. 15, no. 4, pp. 350-361, Apr. 2004.

[22] R. Guérin, J. Rank, S. Sarkar, and E. Vergetis, "Forming connected topologies in Bluetooth ad hoc networks-An algorithmic perspective," presented at the 18th Int. Teletraffic Congr., Berlin, Germany, Sep. 2003.

[23] P. Johansson, M. Kazantzidis, R. Kapoor, and M. Gerla, "Bluetooth: An enabler for personal area networking," IEEE Network, vol. 15, no. 5, pp. 28-37, Sep./Oct. 2001

[24] P. Johansson, R. Kapoor, M. Kazantzidis, and M. Gerla, "Personal area networks: Bluetooth or IEEE 802.11?,' Int. J. Wireless Inf. Netw., vol. 9 , no. 2, Apr. 2002.

[25] Y. Liu, M. Lee, and T. Saadawi, "A Bluetooth scatternet-route structure for multihop ad hoc networks," IEEE J. Sel. Areas Commun., vol. 21, no. 2, pp. 229-239, Feb. 2003.

[26] J. Zheng and M. Lee, "Will IEEE 802.15.4 make ubiquitous networking a reality?: A discussion on a potential low power, low bit rate standard," IEEE Commun. Mag., vol. 42, no. 6, pp. 140-146, Jun. 2004.

[27] T. Salonidis, P. Bhagwat, L. Tassiulas, and R. LaMaire, "Distributed topology construction of Bluetooth personal area networks," in Proc. INFOCOM, Anchorage, AK, Apr. 2001, pp. 1577-1586.

[28] C. Chiasserini, M. Marsan, E. Baralis, and P. Garza, "Toward feasible topology formation algorithms for Bluetooth-based WPANs," presented at the 36th Hawaii Int. Conf. Syst. Sci. (HICSS-36), Big Island, HI, Jan. 2003. 
[29] C. Kalló, C.-F. Chiasserini, R. Battiti, and M. Marsan, "Reducing the number of hops between communication peers in a Bluetooth scatternet," in Proc. IEEE Wireless Commun. Netw. Conf. (WCNC), Atlanta, GA, Mar. 2004, pp. 207-212.

[30] L. Barrière, P. Fraigniaud, L. Narayanan, and J. Opatrny, "Dynamic construction of Bluetooth scatternets of fixed degree and low diameter," presented at the 14th ACM-SIAM Symp. Discrete Algorithms (SODA), Baltimore, MD, Jan. 2003.

[31] M. Marsan, C. Chiasserini, A. Nucci, G. Carello, and L. D. Giovanni, "Optimizing the topology of Bluetooth wireless personal area networks," in Proc. INFOCOM, New York, Jul. 2002, pp. 572-579.

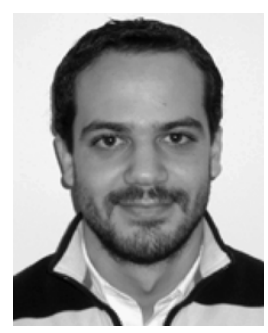

Evangelos Vergetis (S'00) received the B.S. degree in computer science and electrical engineering from Cornell University, Ithaca, NY, in 2001 and the M.S.E. degree in electrical engineering from the University of Pennsylvania, Philadelphia, in 2002. $\mathrm{He}$ is currently working towards the Ph.D. degree in electrical and systems engineering at the University of Pennsylvania.

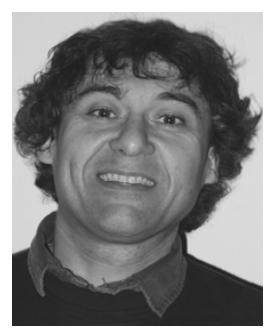

Roch Guérin (F'01) received the Engineer degree from Ecole Nationale Supérieure des Télécommunications (ENST), Paris, France, in 1983, and the M.S. and Ph.D. degrees in electrical engineering from the California Institute of Technology, Pasadena, CA, in 1984 and 1986, respectively.

He joined the Electrical and Systems Engineering Department, University of Pennsylvania, Philadelphia, in 1998, where he is the Alfred Fitler Moore Professor of Telecommunications Networks. Before joining the University of Pennsylvania, he spent over 12 years at the IBM T. J. Watson Research Center, Yorktown Heights, NY, in a variety of technical and management positions. From 2001 to 2004, he was on partial leave from the University of Pennsylvania, starting a company, Ipsum Networks, that pioneered the concept of protocol participation in managing IP networks. He served as the Editor of the ACM SIGCOMM Technical Newsletter, Computer Communication Review (CCR), from 1998 to 2001. $\mathrm{He}$ is on the Technical Advisory Board of France Telecom and Samsung Electronics, and has consulted for numerous companies in the networking area. His research has been in the general area of networking, with a recent focus on developing routing and traffic engineering solutions that are both lightweight and robust across a broad range of operating conditions.

Dr. Guérin received an IBM Outstanding Innovation Award for his work on traffic management in the broad band services network architecture in 1994. He Chaired the IEEE Technical Committee on Computer Communications from 1997 to 1999. He served as Member-at-Large of the Board-of-Governors of the IEEE Communications Society from 2000 to 2002, as General Chair of the IEEE INFOCOM'98 Conference, and as Technical Program Co-Chair of the ACM SIGCOMM 2001 Conference. He was an Editor for the Journal of Computer Networks, the IEEE Communications Surveys, the IEEE/ACM TRANSACTIONS ON NETWORKING, the IEEE TRANSACTIONS ON COMMUNICATIONS, and the IEEE Communications Magazine. He was a Guest Editor for the Special Issue on "Internet QoS" for the IEEE JOURNAL ON SELECTED AREAS IN COMMUNICATIONS published in December 2000.

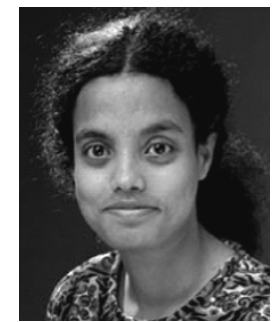

Saswati Sarkar (S'98-M'00) received the M.S.E. degree in electrical communication engineering from the Indian Institute of Science, Bangalore, in 1996 and the Ph.D. degree in electrical and computer engineering from the University of Maryland, College Park, in 2000.

She is currently an Assistant Professor in the Department of Electrical and Systems Engineering, University of Pennsylvania, Philadelphia. Her research interests are in resource allocation and performance analysis in communication networks.

Dr. Sarkar received the Motorola Gold Medal for the Best Masters Student in the Division of Electrical Sciences, Indian Institute of Science and a National Science Foundation (NSF) Faculty Early Career Development Award in 2003. She has been an Associate Editor of IEEE TRANSACTIONS ON WIRELESS COMMUNICATIONS since 2001.

Jacob Rank received the B.S.E. degree in computer and telecommunications engineering from the University of Pennsylvania, Philadelphia, in 2003.

$\mathrm{He}$ is currently working as a Consultant in the Product Engineering Group at CGI-AMS, Fairfax, VA. His research interests include genetic programming and alternative energy sources. 